\title{
ATURUS PRENANTI n. sp. UN NOUVEL HYDRACARIEN DES PYRENEES
}

\author{
par E. ANGEIIER ${ }^{1}$.
}

Aturus prenanti, dédié à Monsieur le Professeur M. Prenant, est une nouvelle espèce d'Hydracarien recueillie à plusieurs reprises dans les Pyrénées. C'est une forme muscicole, sténotherme, qui se rapproche, tant par la morphologie que par l'écologie, du groupe A. spatulifer Piersig, A. elongatus Walter et $A$. comatus Halik. Les caractères sexuels secondaires du $\sigma$ sont cependant assez différents des espèces voisines : bord postérieur du corps presque droit, absence des cupules hyalines postérieures (comme chez $A$. scaber), et surtout les soies postérieures, - qui sont réparties en 2 zones latéro-dorsales bien séparées et appartiennent à plusieurs types.

Mâle. - Couleur jaune-brun. Longueur ventrale : $375 \mu$; longueur dorsale : $340 \mu$; largeur : $305 \mu$. Les bords latéraux du corps sont presque parallèles, de sorte que, - le bord postérieur du corps étant à peu près droit, - l'animal a une forme plus ou moins carrée en vue dorsale. Distance entre les soies antenniformes : $53 \mu$.

La cuirasse dorsale porte 4 paires de pores glandulaires. Les 3 paires antérieures sont situées à peu près sur une même ligne. Chaque pore est accompagné d'une longue soie, large, bifide à son extrémité; la soie externe est nettement plus forte que les 2 autres. La dernière paire de pores est située au quart postérieur du corps; la soie insérée près de chacun d'eux est fine et simple.

L'extrémité postérieure du corps ne présente pas les cupules hyalines qui sont caractéristiques de la plupart des autres Aturus o".

Ia limite postérieure de la cuirasse dorsale est marquée par une rangée de fines soies, pennées, allant du bord latéral du corps à l'échancrure génitale. Entre cette rangée de soies et l'extrémité postérieure se trouve, de chaque côté du corps, une touffe de soies; celles-ci appartiennent à 4 types différents. Les unes sont simples et courtes ( $f i g .1 \mathrm{a}$ ) ; leur longueur moyenne ne dépasse pas 30-35 $\mu$. D'autres (fig. 1b) sont beaucoup plus longues $(80$ à $90 \mu)$, larges, bulbeuses à leur base et recourbées en crochet à leur extrémité; elles sont au nombre de 9 à 12 . Un $3^{\text {e }}$ type (fig. 1c) correspond à

1. Pour les cinquante années de recherches scientifiques du Professeur Marcel Prenant, en hommages respectueux. 


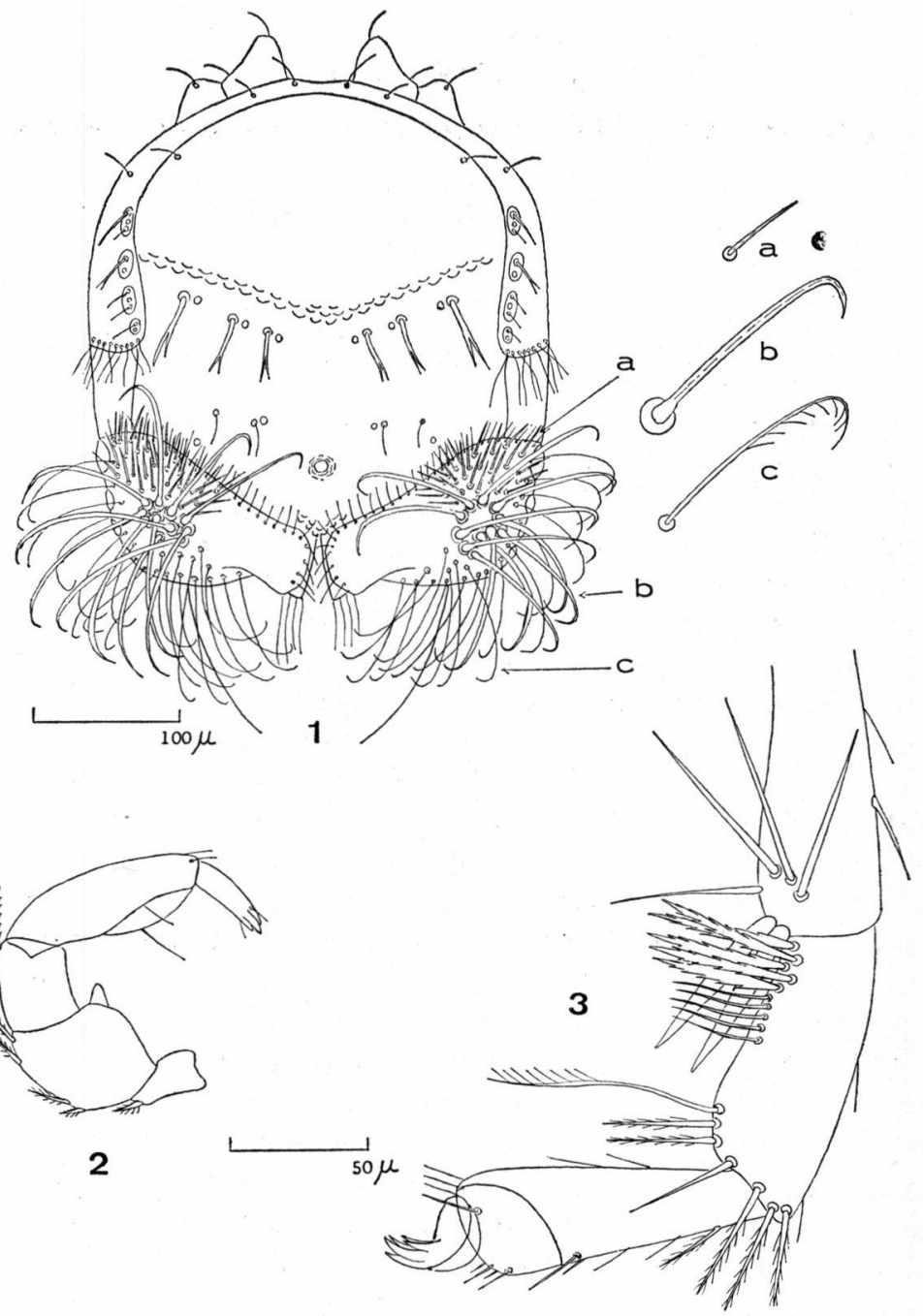

Fig. 1 à 3. - Aturus prenanti n. sp. - $\sigma^{\star} .1$ : face dorsale (a, b et c : types de soies de la région postérieure du corps); 2 : palpe maxillaire; 3 : patte postérieure.

des soies longues (90 à $100 \mu$ ) et fines, recourbées à leur extrémité, et portant une frange de cils. Enfin, sur chaque touffe, une unique soie, fine, est un peu plus longue que les autres $(120 \mu)$.

Organe maxillaire, mandibule et palpe maxillaire ne présentent aucun caractère particulier. Longueur de l'organe maxillaire : $77 \mu$. 
Longueur totale de la mandibule : $100 \mu$ (onglet : $25 \mu$ ). Longueur des articles du palpe maxillaire, sur le bord extenseur (en $\mu$ ) :

$\begin{array}{ccccc}\text { P I } & \text { P II } & \text { P III } & \text { P IV } & \text { P V } \\ 20 & 55 & 34 & 76 & 37\end{array}$

Les épimères couvrent toute la face ventrale du corps. Profondeur du sinus maxillaire : $80 \mu$. Profondeur de l'échancrure génitale : $65 \mu$. Les cupules génitales sont au nombre de 11-12 de chaque côté de l'échancrure génitale. Longueur du support chitineux du pénis : $102 \mu$; envergure des cornes antérieures : $51 \mu$; envergure des cornes postérieures : $103 \mu$.

Longueur des pattes : p $1: 350 \mu ;$ p $2: 390 \mu ;$ p $3: 500 \mu ;$ p 4 : $610 \mu\left(4^{\mathrm{e}}\right.$ article : $102 \mu ; 5^{\mathrm{e}}$ article : $110 \mu ; 6^{\mathrm{e}}$ article : $\left.108 \mu\right)$. Les griffes, élargies à leur base, présentent chacune 2 dents accessoires. Le $5^{\mathrm{e}}$ article des p 3 porte une longue soie dans la région médiane. Les caractères essentiels des p 4 sont les suivants :

$4^{\mathrm{e}}$ article : à l'extrémité distale sont insérées, sur le bord fléchisseur, 2 longues lames chitineuses rappelant $A$. villosus ou $A$. elongatus; à côté se trouvent 3 longues soies, fortes, accompagnées d'une autre plus fine.

$5^{\text {e }}$ article : la partie proximale porte 5 larges soies pennées, suivies, sur le bord fléchisseur, de 5 soies simples. L'extrémité distale de l'article présente une longue soie pennée, 2 soies pennées plus courtes et 1 soie simple, - sur le bord fléchisseur; 3 soies pennées sur le bord extenseur.

Cette nouvelle espèce, sténotherme, a été recueillie dans les mousses de plusieurs stations du ruisseau de l'Orle, affluent rive droite du Lez (Ariège), entre 650 et $1500 \mathrm{~m}$ d'altitude. Elle est également fréquente dans les affluents de la Neste d'Aure (Hautes-Pyrénées).

Elle est toujours associée à plusieurs autres espèces d'Aturus, A. scaber, A. crinitus, A. villosus, A. gallicus ou A. elongatus processiger. Dans l'état actuel de la systématique du genre Aturus, il n'est pas possible de déterminer rigoureusement les $q$, et par conséquent de savoir avec certitude quelle était la $q$ d'Aturus prenanti.

\section{Remarques sur les Aturus des Pyrénées.}

11 espèces du genre Aturus sont actuellement connues dans les Pyrénées françaises :

A. scaber Kramer 1875

A. crinitus Thor 1902

A. villosus Motas \& Soarec 1939

A. elongatus processiger Lundblad 1956

A. protzi Piersig 1901. - Syn. : A. scitulus Angel. 1950; A. natangensis Angel. 1931 (non $A$. natangensis Protz). 
A. intermedius Protz 1900

A. gallicus Viets 1939

A. prenanti n. sp.

A. barbatulus Viets 1936

A. brachypus Viets 1936. -- Syn. : A. paucisetus Motas \& Tanasachi 1946.

A. karamani Viets 1936. - Syn. : A. pauciporus Walter 1947.

Aturus karamani est une forme des eaux interstitielles. Les 10 autres espèces vivent dans les eaux courantes et sont essentiellement muscicoles.

A. brachypus est relativement rare; en France, il est connu des Pyrénées-orientales (vallée du Tech) et du Tarn (ruisseau du Céret).

Le peuplement en Aturus des eaux de basse et moyenne altitude, sur l'ensemble de la chaîne des Pyrénées, est essentiellement formé par :
A. scaber
A. intermedius
A. protzi
A. barbatulus

A. scitulus, déerit par C. Angelier [1950], est synonyme de $A$. protzi. A. scaber et $A$. intermedius ont une large répartition en Europe. A. protzi est signalé en Allemagne, Belgique, Espagne, Massif Central, Corse et Pyrénées; il est probablement aussi répandu que les 2 autres espèces

Quant à $A$. barbatulus, c'est une forme à dispersion beaucoup plus restreinte, limitée à l'Europe méridionale (Yougoslavie, Corse, Pyrénées).

Dans les eaux courantes de haute altitude, A. scaber devient plus rare; les espèces essentielles sont :
A. crinitus
A. gallicus
A. villosus
A. prenanti
A. elongatus processiger

A. crinitus est répandu dans les eaux froides de toute l'Europe. Les 4 autres espèces ou sous-espèces sont par contre, - dans l'état actuel de nos connaissances, - des endémiques pyrénéens.

$A$. villosus est assez proche d'A. crinitus; ils peuvent être associés (ruisseau de l'Orle, Ariège). A. elongatus processiger est une sousespèce décrite des Pyrénées espagnoles par $O$. Lundblad [1956], nouvelle pour la faune française; l'espèce $s$. str. n'a jamais été signalée dans les Pyrénées. A. gallicus, décrit par K. Viets [1939] de la vallée d'Ossau (Basses-Pyrénées), a été retrouvé dans l'Ariège (ruisseau de l'Orle); très proche d'A. asserculatus Walter 1906, encore inconnu dans les Pyrénées, - il n'en est peut-être qu'une sous-espèce. Enfin, A. prenanti a été recueilli dans l'Ariège et les Hautes-Pyrénées. 
Il est intéressant de noter que sur 5 espèces d'Aturus sténothermes des Pyrénées, 4 sont des endémiques, et 3 de celles-ci sont étroitement apparentées à des formes alpines. L'isolement des populations sténothermes alpines et pyrénéennes, après les glaciations, a conduit à un endémisme élevé dans les Aturus de haute altitude. On retrouve d'ailleurs des exemples analogues dans d'autres genres d'Hydracariens (Feltria bispinosa Angel. 1950, très répandu dans les eaux froides des Pyrénées centrales, est sans doute une forme dérivée de Feltria romijni Bessel. 1930, absent lui-même des Pyrénées).

\section{Aturus prenanti n. sp., new Hydrachnellae (Acari) of the Pyrenees}

The author describes a new species of Hydrachnellae, and summarizes our knowledge of the Aturus in the French Pyrenees.

Aturus prenanti n. sp., eine neue Hydrachnellae (Acari) aus den Pyrenäen

Der Verfasser beschreibt eine neue Art der Hydrachnellae und gibt im folgenden den Stand der Forschung über die Gattung Aturus in den französischen Pyrenäen.

\section{BIBLIOGRAFHIE}

Angelier (C.). 1931. - Contribution à l'étude de la faune hydracarienne de la Marne. Trav. Labor. Hydrobiol. Piscicult. Univ. Grenoble, 23 : 83-136.

Angelier (C.). 1949. - Hydracariens nouveaux des Pyrénées ( $3^{e}$ note) : Aturus barbatulus Viets 1936, nouveau pour la faune française. Bull. Mus. Hist. Nat., Paris, (2), 21, 6 : 697-699.

Angelier (C.) . 1950. - Hydracariens nouveaux des Pyrénées (4 note). Bull. Mus. Hist. Nat., Paris (2), 22, 1 : 81-84.

Angelier (E.), Decamps (H.) \& Rey (J.). 1963. - Les Hydracariens du Céret : étude systématique et écologique. Bull. Soc. Hist. Nat. Toulouse, $98,3 / 4: 459-500$.

I.undalad (O.). 1956. - Zur Kenntnis süd- und mitteleuropäischer Hydrachnellen. Ark. Zool., Slockholm, (2), 10, 1 : 1-306.

Motas (C.) \& SOAREC (J.). 1939. - Sur deux nouveaux Hydracariens français recueillis dans les Pyrénées. Ann. Sci. Univ. Jassy, 25, $2: 1-13$.

Viets (K.). 1939. -- Wassermilben (Hydrachnellae und Porohalacaridae, Acari) aus den französischen Pyrenäen. Zool. Anz., 125, 1/2 : 1-15.

(Station Biologique du lac d'Orédon; Laboratoire de Zoologie de la Faculté des Sciences, Toulouse.) 